\title{
Wireless Antenna Detection of Electrostatic Discharge Events
}

\author{
Allen Andersen, Member, IEEE, and JR Dennison, Member, IEEE
}

\begin{abstract}
Wireless intra-spacecraft communication technology is being developed for signal transfer on space missions to save weight and simplify design. One consideration for this new technology is its interaction with space environment-induced electrostatic discharges (ESD). The short time scales of spacecraft ESD events results in broad frequency band signals that can interact with high frequency wireless antennas. These interactions present a source of signal noise. However, they also present a possibility of in-flight wireless ESD monitoring.

We present laboratory measurements of arcing on common spacecraft insulators using commercially available single band 2.4 $\mathrm{GHz}$ and dual band 2.4/5.8 GHz Wi-Fi antennas. These wireless detections are shown to coincide with direct simultaneous measurements of discharge event currents. The amplitudes of the wireless signals are shown to provide a measure of the relative magnitude of the arcs, although the narrow frequency ranges of Wi-Fi antennas make absolute measures of the discharge intensity impractical. The wireless signals are shown to provide very accurate nsec timing of ESD events, which presents the potential of identifying arc location on a spacecraft with the use of time-offlight detection from multiple antennas.
\end{abstract}

Index Terms-Antenna, arcing, breakdown, electrostatic discharge (ESD), polymers, space environment effects, spacecraft charging, standards.

\section{INTRODUCTION}

$\mathrm{W}$ ireless intra-spacecraft communication systems have been proposed due to their potential for weight-saving and design flexibility compared to wired systems [1,2]. Such systems are essentially an on-board Wi-Fi network allowing various spacecraft systems to communicate wirelessly. Electrostatic discharge (ESD) is known to be the primary cause of spacecraft failures and anomalies due to their interactions with the space environment [3, 4]. ESD are produced in spacecraft materials as accumulated charge from the space plasma environment produces an electric field that exceeds a material's dielectric breakdown field strength.

In this paper we consider the possible interactions of wireless antenna systems with ESD. The short time duration of many ESD events results in broad frequency spectra of radiated emissions. Such emissions will be a source of instrument noise, which can potentially disrupt measurements and communications. However, this research focuses primarily on the concept of in-flight ESD condition monitoring using spacecraft antenna.

\footnotetext{
Manuscript received September, 2018. The work of A. Andersen was supported by the NASA Space Technology Research Fellowship.

Allen Andersen was a graduate student at Utah State University, Logan, UT 84322 USA. He is now with the Jet Propulsion Laboratory, Pasadena, CA 91109 USA (e-mail: allen.j.andersen@jpl.caltech.edu).
}

Except in the few missions that fly dedicated ESD monitors (e.g., refs [A] and [B]), ESD are typically only identified after an anomaly occurs. An increased awareness of ESD eventsbenign or otherwise - and information about their occurrence, timing, and location will enhance predictions of the risk associated with problematic ESD for future missions. Also, inflight ESD monitoring could result in the identification of ESD prone areas of a spacecraft before anomalies or permanent damage occurs. Depending on the configuration of a spacecraft, such advanced early warning may make it possible to identify systems to be put in a protected state in response to observations of increased ESD activity

We propose that intra-spacecraft wireless communication antennas are capable of in-flight ESD monitoring and that-if multiple antennas with sufficient time resolution are used - one can detect not only if and when ESD occur, but where ESD occur using simple time-of-flight calculations. Such systems may require little, if any, additional hardware on the spacecraft, if spacecraft are intelligently designed a priori to monitor for ESD signatures on existing antenna incorporated for other communication purposes.

\section{Proof OF CONCEPT Methods}

We present ground-based measurements of ESD using offthe-shelf Wi-Fi antennas as proof of concept to encourage further development of in-flight wireless ESD monitoring systems. First, we present evidence of detection of ESD using a Wi-Fi antenna in coincidence with simultaneous leakage current measurements taken with standard ammeters. Second, we present examples of ESD position determination using multiple Wi-Fi antennas. Such location methods are obviously similar to standard GPS applications, which use similar GHz frequency signals [D]. We note that similar methods have been developed for detecting discharges in terrestrial applications, such as high voltage DC power transmission or consumer electronics [5-8]. However, in this paper we demonstrate that the methods are still effective when using cost-effective, standard, off-the-shelf Wi-Fi antennas rather than antennas that are specifically designed for identifying ESD. Note that the bandwidth limitations of Wi-Fi antennas make interpretations of ESD signal spectra and amplitude less reliable.

JR Dennison is with the Materials Physics Group in the Physics Department, Utah State University, Logan, UT 84322 USA. (e-mail: JR.Dennison@usu.edu).

Color versions of one or more of the figures in this paper are available online at http://ieeexplore.ieee.org.

Digital Object Identifier 


\section{A. Coincidence Measurements of Discharge Events}

The Utah State University Materials Physics Group (MPG) measures the likelihood of dielectric breakdown of insulating materials using an in vacuo parallel plate voltage step-up to breakdown method [9]. This method is adapted from the method recommended in spacecraft charging and ASTM standards, the details of which have been published previously [9-13]. In summary, leakage current is measured as voltage is applied across a dielectric thin-film sample; both transient partial discharges and total dielectric breakdown are observed. In addition to the typical test setup, a $2.4 \mathrm{GHz} \mathrm{Wi}-\mathrm{Fi}$ antenna, placed external to a vacuum chamber adjacent to standard glass viewport, was connected to a $50 \Omega$ load and monitored using a digital storage oscilloscope (Tektronix TDS 2014) with custom LabVIEW data acquisition software.

The results of a typical test are shown in Fig. 1. The ammeter (Amprobe, 38XR-A), with $100 \mathrm{nA}$ resolution at $2 \mathrm{~Hz}$ acquisition rate, is not as sensitive as the antenna but does provide reliable arc amplitude information. However, discharges seen by the ammeter are found to temporally correlate to events seen in by the antenna. Larger-amplitude microammeter events were shown to correspond to current integrated over multiple separate fast discharges observed with the oscilloscope. Discharges are observed until complete breakdown creates an electrical short as seen in Fig. 1 beginning at $\sim 1800 \mathrm{~s}$, where the steadily increasing solid blue markers correspond to an ohmic breakdown current set by current limiting resistors in the system [9]. Following complete breakdown, the samples no longer produces transients, as confirmed by the lack of antenna events.

Other experiments used Wi-Fi antennas in similar configurations to monitor the timing of arc events $[\mathrm{C}]$ produced by electron fluxes incident on dielectric materials in a space simulation test chamber [E]. Wi-Fi antenna were placed inside a standard UHV system and connected via standard RF SMA vacuum feedthroughs to a $500 \mathrm{MS} / \mathrm{s}$ digital storage oscilloscope (LeCroy 9350AM). Simultaneous arc signals were measured with lower resolution power supply integral current and voltage monitoring (Hewlett Packard HP6024A with $\sim 1 \mathrm{~s}$ acquisition time), somewhat faster higher-precision electrometers (Keithley 199 with $100 \mathrm{nA}$ resolution and $100 \mathrm{~ms}$ acquisition time and Keithley 6517B with $<1 \mathrm{fA}$ resolution and $15 \mathrm{~ms}$ acquisition time a $1 \mathrm{nA}$ ), current signals across a $50 \Omega$ shunt load and monitored using a digital storage oscilloscope (Tektronix TDS 2014 with $\sim 100 \mathrm{nA}$ resolution and $\sim 40 \mathrm{~ms}$ collection circuit RC time constant), and 30-60 fps video [C]. Measurements confirmed coincidence of the ESD events to with the resolution of the various detection methods. The WiFi antenna provided the best temporal resolution, on the order of $10 \mathrm{~ns}$.

This result confirms that a typical Wi-Fi antenna is capable of detecting discharges and timing them with $10^{-9} \mathrm{~s}$ to $10^{-6} \mathrm{~s}$ precision, set by the sampling rate of the detecting oscilloscope. By contrast, direct current measurements with standard microammeters or electrometers probing the leakage current are limited in time resolution by the instrument acquisition times of $10^{-2} \mathrm{~s}$ to $1 \mathrm{~s}$, while current measurements through a shunt resistor using a digital oscilloscope has temporal resolution set

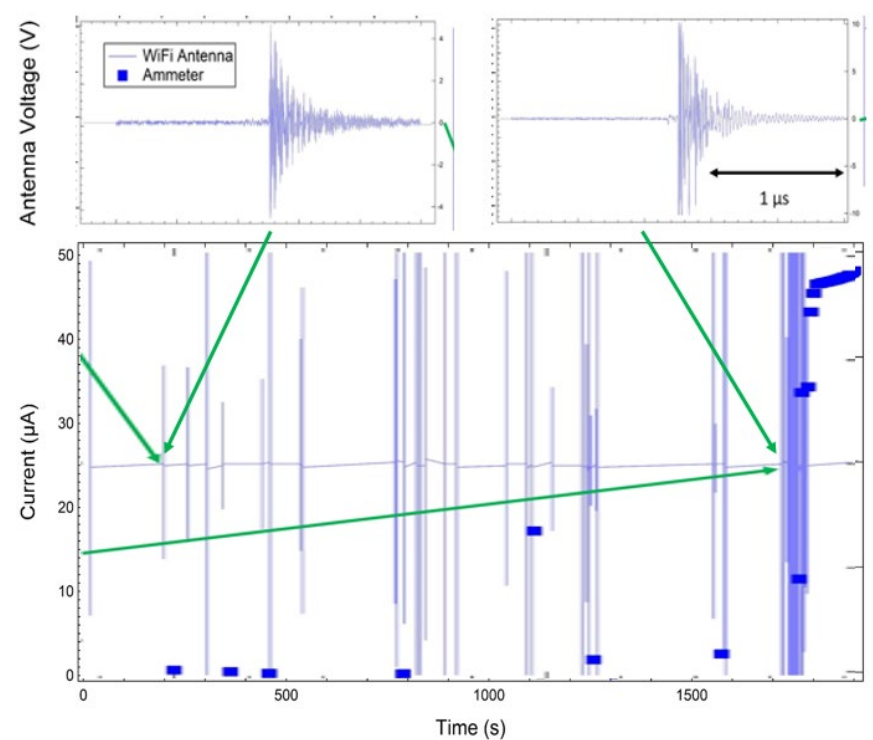

Figure 1. Partial discharges measured during a voltage step-up test of biaxiallyoriented polypropylene (BOPP) [9]. The primary graph shows the large amplitude current spikes versus elapsed time during the voltage ramp, as measured with the slower microammeter. The insets show two examples of individual trigger events measured with a $2.4 \mathrm{GHz}$ WiFi antenna connected to a $50 \Omega$ load oscilloscope shunt.

by the RC time constant of the collection circuit ( $\sim 40 \mathrm{~ms}$ for our setup) [9]. Tests are in progress to determine the effects of using lower frequency broad band dectection Rogowski coils (Power Electronics Measurements CWT MiniHF 015 Current probe with $150 \mathrm{~Hz}$ to $30 \mathrm{M} \mathrm{Hz}$ response range).

\section{B. Spatial Localization of Discharge Events}

Having confirmed that Wi-Fi antennas can demonstrate if and when ESD occur, we now discuss how to determine where they occur using differences in time-of flight measurements. The spatial resolution of the calculated ESD location depends on the time resolution of the measurements. That is, the smallest resolved difference in time between signals received by different antennas, $\Delta t_{\text {min }}$, corresponds to the minimum spatial difference that can be distinguished

$$
\Delta x_{\min }=c \Delta t_{\min }
$$

where $c$ is the speed of light. This corresponds to a nominal lower bound on spatial detection of $30 \mathrm{~cm}$ and $1.5 \mathrm{~cm}$ for $1 \mathrm{GS} / \mathrm{s}$ $\left(\Delta t_{\min }=1 \mathrm{~ns}\right)$ and $20 \mathrm{GS} / \mathrm{s}\left(\Delta t_{\min }=50 \mathrm{ps}\right)$ oscilliscopes, respectively.

In principle, it is possible to improve resolution by fitting the measured data points of the oscilloscope trace, allowing interpolation between data points. A common model envelope fitting function for the measured current traces is

$$
\begin{aligned}
& I_{\text {arc }}\left(t ; t_{\text {peak }}, I_{\text {peak }}, \tau_{\text {rise }}, \tau_{\text {fall }}, \omega, \phi\right)= \\
& \quad I_{\text {peak }} \cdot\left\{\sin \left[\omega\left(t-t_{\text {peak }}\right)+\phi\right]\right\} . \\
& \quad\left\{\exp \left[-\left(t-t_{\text {peak }}\right) / \tau_{\text {rise }}\right]-\exp \left[-\left(t-t_{\text {peak }}\right) / \tau_{\text {fall }}\right]\right\} .
\end{aligned}
$$

Here, time-of flight analysis is determined by comparison of the time, $t_{\text {peak }}$, at peak amplitude $I_{\text {peak }} . \phi$ is the phase shift of the signal at the peak amplitude. $\omega$ is the frequency of the 

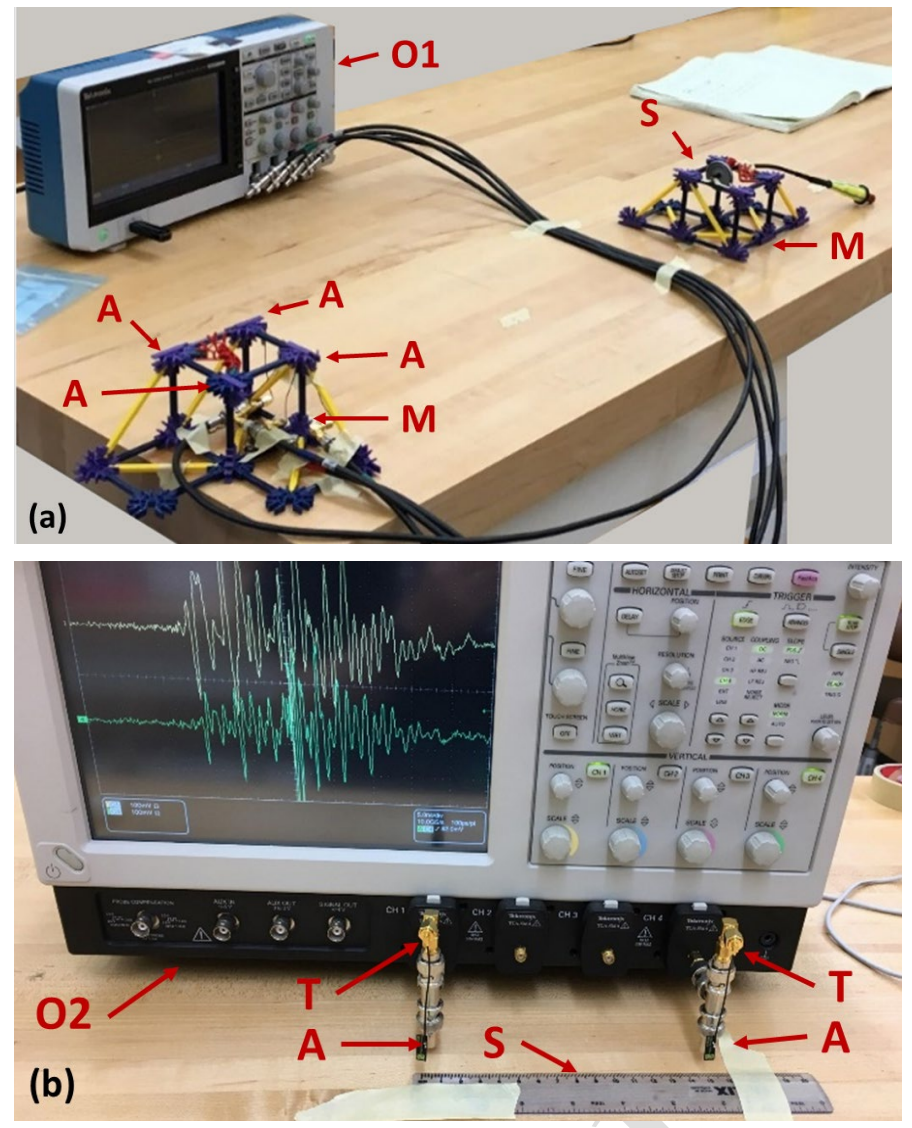

Figure 2. Examples of tabletop ESD detection setups using off-the-shelf WiFi antennas. (a) Wi-Fi antenna characterization setup configuration using four antennas and a $1 \mathrm{GS} / \mathrm{s}$ oscilloscope. (b) Difference in time-of-flight setup using two antennas and a $20 \mathrm{GS} / \mathrm{s}$ digital oscilloscope. Oscilloscopes (O1 and O2), antennas (A), antenna mounts (M), arc source (S), and $50 \Omega$ terminators (T) are identified.

measured signal, which can be determine precisely using a FFT of the current traces. $\tau_{\text {rise }}$ and $\tau_{\text {fall }}$ are the arc envelope rise and fall times, found by fitting the envelope. Real time fits to arc traces acquired with computer under LabView control have been implemented in our arc detection chamber [C].

Difficulties in identifying matching features in signals resulting from differences in antenna or cabling response to the ESD signal, polarization effects, etc., may introduce additional uncertainty in $\Delta t_{\min }$ beyond the maximum time resolution of the oscilloscope [8]. Obviously, the spatial resolution might also be limited by the spatial extent of the antennas or the arc sources themselves. For the tests outlined in this section, standard dual band Wi-Fi antennas (Taoglas FXP840 Freedom Series Super Small Monopole Dual-band 2.4 GHZ and 4.9-6 $\mathrm{GHz}$ antennas) were used due to their smaller form factor. These antennas are $14 \times 5 \times 0.1 \mathrm{~mm}$ and are designed for Wi-Fi or Bluetooth type communications for tablet or smartphone sized devices. A hand held piezoelectric arc source (Educational Innovations Piezo Igniter HS-2B) attached to a small spark gap produced arcs localized to $\sim 1 \mathrm{~mm}^{3}$.

To test the response of these antennas, four off-the-shelf WiFi antennas were connected to a $1 \mathrm{GS} / \mathrm{s}$ digital oscilloscope (Tektronix TBS 2000 Series) with identical cabling, coaxial connectors and $50 \Omega$ terminators. They were placed at known distances from a controlled piezoelectric spark source for the
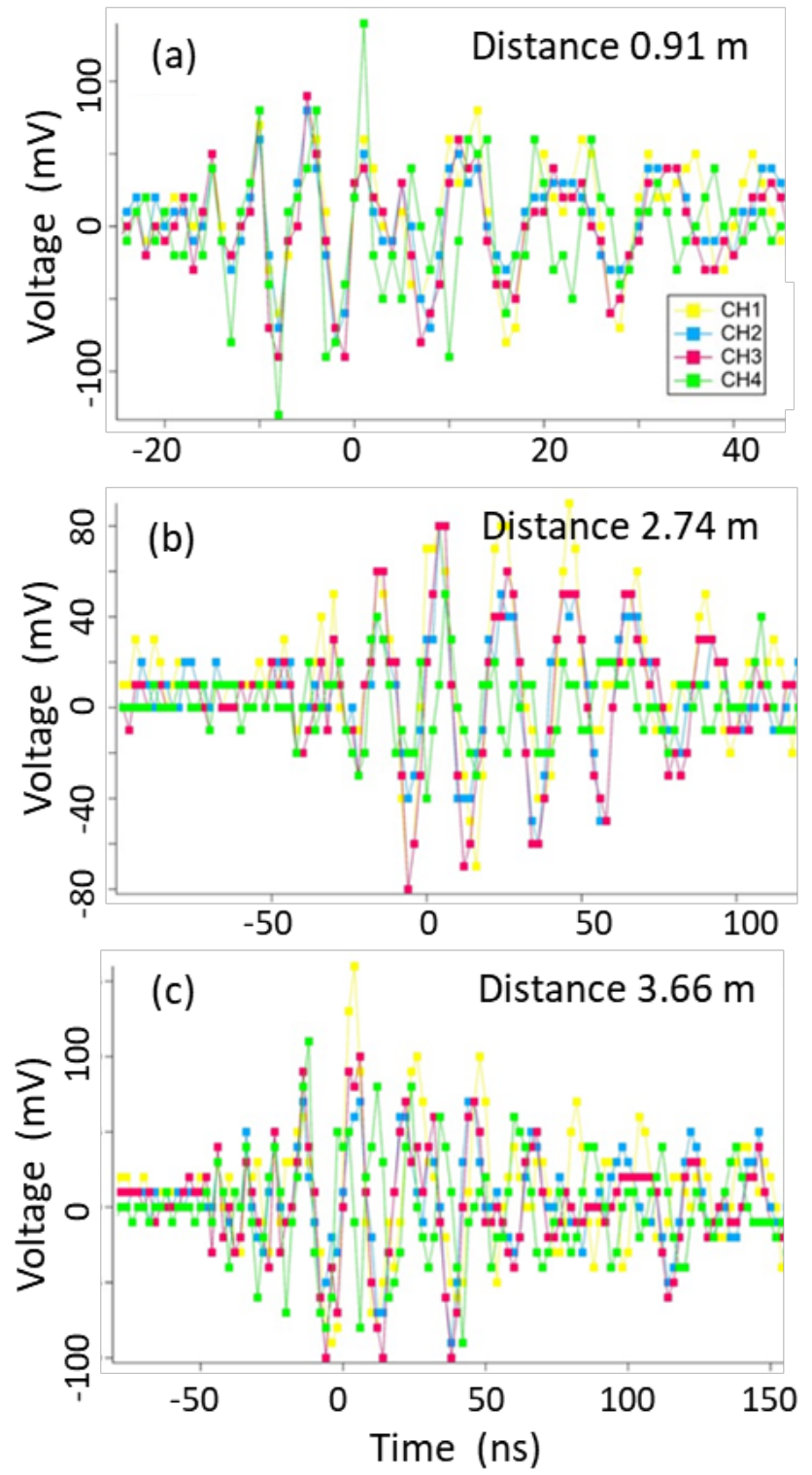

Figure 3. Traces from antenna characterization tests, each corresponding a discharge event as seen by four antennas at three different fixed distances [see Fig 2(a)]. Data were acquired at $1 \mathrm{GS} / \mathrm{s}$.

tabletop ESD detection setup shown in Fig. 2(a). Both the antenna and the piezoelectric arc source were held in place using small structures made from K'NEX building toys. The responses of each antenna to were compared for several discharge events. In agreement with other published results, it was observed that the first few oscillations of the signal correlated very well in phase from antenna to antenna then gradually went out of phase from one another [8]. For this reason, the first peak above the noise was chosen as the feature from which to extract time-of-flight differences in subsequent tests.

Figure 3 shows three typical sets of current traces from such antenna characterization tests, each corresponding a discharge event as seen by four antennas at three different fixed distances [see Fig 2.(a)]. The first few oscillations in each channel are in phase before a relative phase shift occurs between each pulse.

The simplest case for localization is the one-dimension case with the source and detectors co-linear-by simply measuring the difference in detection times for discharges originating 

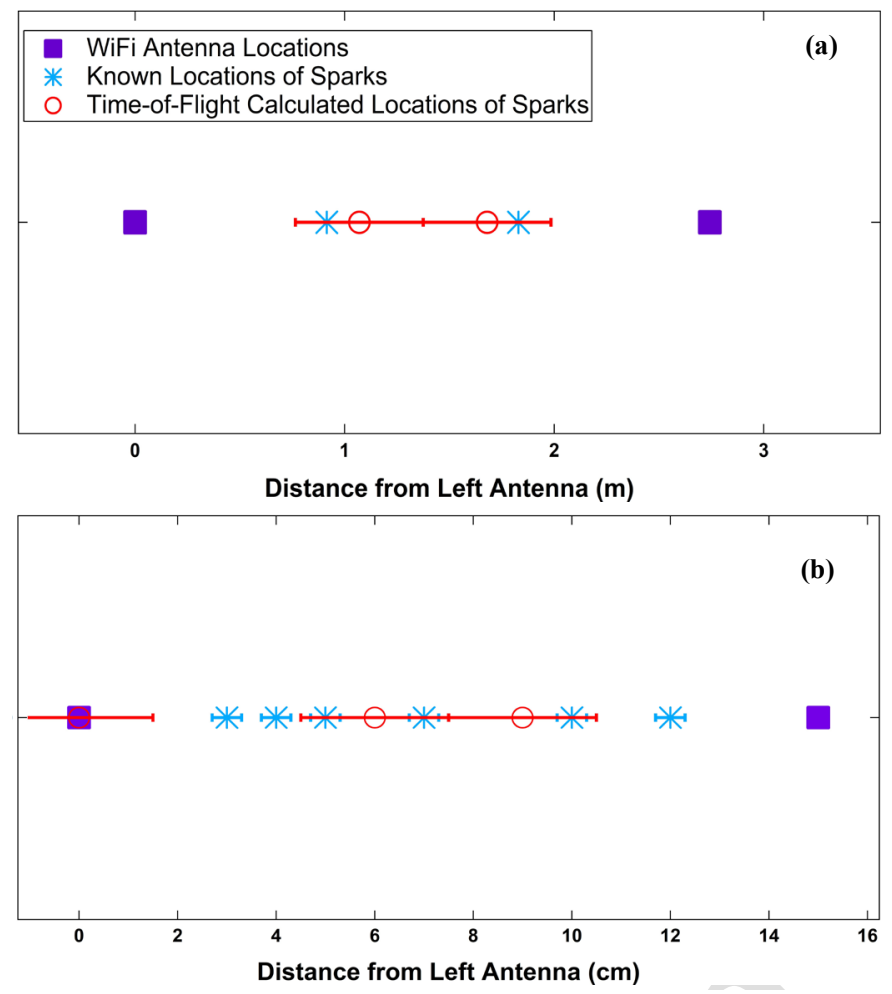

Figure 4. Time of flight difference calculated locations of ESD compared to known locations and Wi-Fi antenna locations with a $1 \mathrm{GS} / \mathrm{s}$ oscilloscope (a) and a $20 \mathrm{GS} / \mathrm{s}$ scope (b). Error bars are estimated uncertainties based on the expected best resolution for each oscilloscope, based on their sample rate.

between two antennas. A discharge outside the antennas in this geometry would not yield its location, only the distance between the two antennas. Given a known separation between two antennas, $l$, and defining the left antenna to be located at the origin, the location $x$ of a discharge is

$$
x=\frac{l-c \Delta t}{2}
$$

for $\Delta t \equiv t_{l}-t_{r}$ where $t_{l}$ and $t_{r}$ are the times of matched pulse features as seen by the left and right antennas, respectfully. It is straightforward to generalize time-of-flight calculations to three dimensions. Indeed, such setups have been used in terrestrial applications [8].

For the first location tests, pairs of antennas were placed at $2.74 \mathrm{~m}$ apart with one discharge at $0.91 \mathrm{~m}$ and another at 1.83 $\mathrm{m}$. Given the time resolution of the $1 \mathrm{GS} / \mathrm{s}$ oscilloscope, a best case of $0.3 \mathrm{~m}$ resolution was expected. Fig. 4(a) shows that indeed the setup can locate ESD to within the expected resolution.

For the second tests a faster, $20 \mathrm{GS} / \mathrm{s}$ digital oscilloscope (Tektronix TDS7704B) was used, with an expected resolution of $1.5 \mathrm{~cm}$ as shown in Fig. 2(b). In this test, the antennas were spaced $15 \mathrm{~cm}$ apart with discharges set off at 3, 4, 5, 7, 10, and $12( \pm 0.3) \mathrm{cm}$ from the left antenna to determine how well the setup could discriminate between different locations. With the faster oscilloscope the signals were more sensitive to phase differences between signals (see Fig. 5) and the discharges at 4, 5,7 , and $10 \mathrm{~cm}$ were not distinguishable. In this case, the discrepancies of the calculated locations in Fig. 4(b) were on the order of $\pm 3 \mathrm{~cm}$, about twice the expected uncertainty based

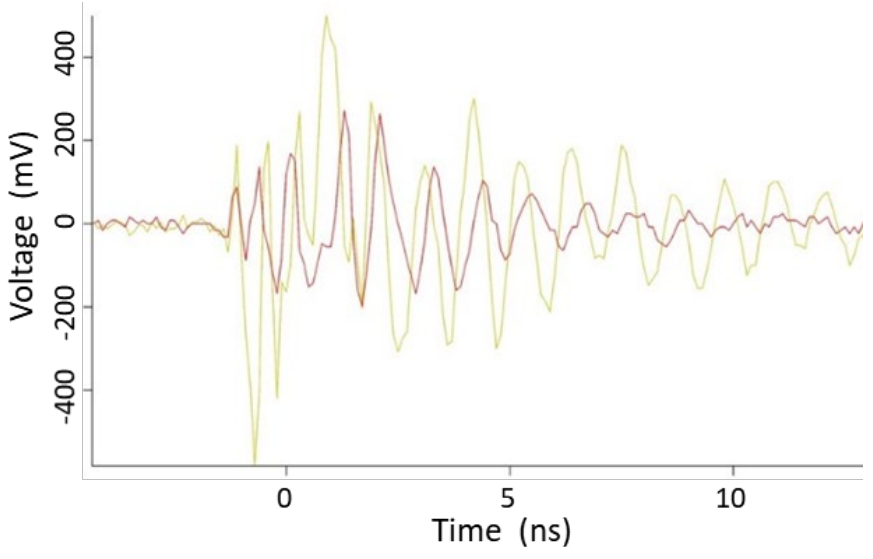

Figure 5. RF traces from a discharge measured using the $20 \mathrm{GS} / \mathrm{s}$ digital oscilloscope [see Fig. 2 (b)].

solely on the oscilloscope sampling rate. Matching features was much more difficult for this oscilloscope since a fairly consistent attribute of the traces is that, when there seem to be corresponding features, the shifting in the relative phase interferes with the timing. Picking the first features no longer gives expected locations within uncertainty. Even without achieving the expected resolution, using the faster oscilloscope resulted in about an order of magnitude improvement in resolution compared to the first measurement.

\section{CONCLUSION}

The ground-based tests presented here demonstrate that standard cost-effective off-the-shelf Wi-Fi antennas are well suited for detecting ESD events, timing their occurrence, and that when multiple antennas at known locations are used differences in times-of-flight can be used to locate discharges spatially. Both 2D and 3D spatial determinations are possible and are enhanced by use of multiple detectors, in analogy with standard GPS signal processing. The spatial resolution of ESD location setups depends on the time resolution of the instruments receiving the RF signal, but can also be limited by any distortions the signal pulse shape. Both spatial and temporal resolution can in principle be enhanced with the use of more sophisticated signal processing which is a wellestablished technology.

As Wi-Fi-like intra-spacecraft communications become more common, such systems could be used to monitor ESD events during spaceflight with minimal additional complexity and expense.

\section{ACKNOWLEDGEMENT}

We gratefully acknowledge contributions from other members of the Materials Physics Group and helpful conversations with Pablo Narvaez of JPL. This work was supported by a NASA Space Technology Research Fellowship.

\section{REFERENCES}

W. H. Zheng, and J. T. Armstrong, "Wireless intra-spacecraft communication: the benefits and the challenges," in Proc. 2010 NASA/ESA Conf. on Adaptive Hardware and Systems (AHS), Anaheim, CA Jun. 2010, pp. 75-78. 
S. Yoshida, N. Hasegawa, Y. Kobayashi, A. Miyachi, H. Sakaki, K. Nishikawa, Y. Moriguchi, S. Furuta, C. Maekawa, and I. Urushibara, "Wireless sensor network system with wireless powering by time division operation at $5.8 \mathrm{GHz}$ in a reusable rocket," in Proc. IEEE International Symp. On Radio Frequency Technol. (RFIT), Sendai, Japan Aug. 2015 pp. 232-234.

C. C. Reed, R. Briët, and M. Begert, "ESD Detection, Location and Mitigation, and Why they are Important for Satellite Development," in Proc. 13 ${ }^{\text {th }}$ Spacecraft Charging Technol. Conf., Pasadena, CA, 2014, pp. 1-6.

D. C. Ferguson, S. P. Worden, and D. E. Hastings, "The Space Weather Threat to Situational Awareness, Communications, and Positioning Systems," IEEE Trans. on Plasma Sci., vol. 43, no. 9, pp. 3086-3098, 2015.

Z. Tang, C. Li, X. Cheng, W. Wang, J. Li, and J. Li, "Partial discharge location in power transformers using wideband RF detection," IEEE Trans. on Dielectr. and Electr. Insul., vol. 13, no. 6, pp. 1193-1199, 2006.

A. H. El-Hag, N. Qaddoumi, R. Mourtada, E. A. Murawwi, A. Nimer, K. AlMazam, M. Hirzallah, and A. Huwair, "Multi-purpose RF antenna for partial discharge and oil quality monitoring," in Proc. $3^{\text {rd }}$ International Conf. on Electr. Power and Energy Conversion Systems, Istanbul, Turkey Oct. 2013, pp. 1-5.

J. Liu, G. Zhang, J. Dong, and J. Wang, "Study on miniaturized UHF antennas for partial discharge detection in high-voltage electrical equipment," Sensors, vol. 15, no. 11, pp. 29434-29451, 2015.

8] R. A. Oglesbee, "Spatial Location of Electrostatic Discharge Events Within Information Technology Equipment," M.S. thesis, Electr. Eng. Univ. of Kentucky, Lexington, KY, 2007.

9] A. Andersen, "The Role of Recoverable and Non-recoverable Defects in DC Electrical Aging of Highly Disordered Insulating Materials," PhD dissertatoin, Depart. of Physics, Utah State Univ., Logan, UT, 2018.

Mitigating in space charging effects-a guideline, document NASAHDBK-4002A, 2011.

Standard Test Method for Dielectric Breakdown Voltage and Dielectric Strength of Solid Electrical Insulating Materials Under Direct-Voltage Stress, document ASTM D3755-14, 2014.

A. Andersen, J. R. Dennison, and K. Moser, "Perspectives on the Distributions of ESD Breakdowns for Spacecraft Charging Applications," IEEE Trans. on Plasma Sci., vol. 45, no. 8, pp. 20312035, 2017.

A. Andersen, and J. R. Dennison, "An Enhanced Operational Definition of Dielectric Breakdown for DC Voltage Step-up Tests." in Proc. IEEE Conf. Electr. Insul. Dielectr. Phenomena (CEIDP), Fort Worth, TX, Oct. 2017, pp. 433-436.
[A] CREES ref, Fredrickson

[B] Japan microsatelitte, Mingo Cho 2013

[C] J. R. Dennison, G. Wilson, J. Mojica Decana and B. Wood, "Real-time Video Processing of Arcing Events to Determine Coincidence," American Physical Society Four Corners Meeting, University of Utah, Salt Lake City, UT, October 12-13, 2018.

[D] http://www.navcen.uscg.gov/pubs/gps/gpsuser/gpsuser.pdf "NAVSTAR GPS User Equipment Introduction" (PDF). US Government. Retrieved 2013-07-24.

[E] R. H. Johnson, L. D. Montierth, J. R. Dennison, J. S. Dyer, and E. Lindstrom, "Small Scale Simulation Chamber for Space Environment Survivability Testing," IEEE Trans. on Plasma Sci., 41(12), 2013, 34533458.

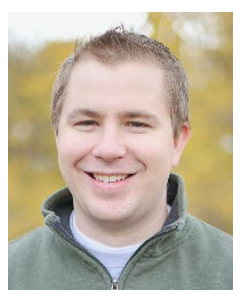

Allen Andersen (GS'15) was a graduate student at Utah State University in Logan, UT where he received his PhD in physics in 2018. He received a BS degree in physics from BYU-Idaho in 2012. He is now with the Jet Propulsion Laboratory Natural Space Environments group. His research area includes electrostatic discharge and electron transport in dielectric mateirals with an emphasis in spacecraft charging effects. IEEE Graduate Student Member.

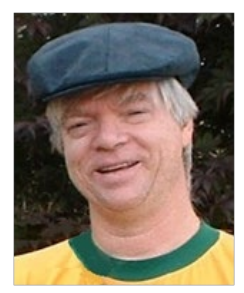

JR Dennison (M'12) received the B.S. degree in physics from Appalachian State University, Boone, NC, in 1980, and the M.S. and Ph.D. degrees in physics from Virginia Tech, Blacksburg, in 1983 and 1985, respectively. He was a Research Associate with the University of MissouriColumbia before moving to Utah State University (USU), Logan, in 1988. He is currently a Professor of physics at USU, where he leads the Materials Physics Group. He has worked in the area of electron scattering for his entire career and has focused on the electron emission and conductivity of materials related to spacecraft charging for the last three decades. IEEE member. 